\title{
Fatores de risco associados à saúde dos dentistas - uma abordagem epidemiológica
}

\author{
Klaus Loges (UNISINOS) kloges@unisinos.br \\ Fernando Gonçalves Amaral (PPGEP/UFRGS) amaral@producao.ufrgs.br
}

\begin{abstract}
Resumo
A odontologia é uma profissão que impõe ao seu praticante uma série de fatores capazes de alterar as condições de trabalho do ponto de vista sócio-psico-fisiológico e organizacional. Este estudo tem como objetivo contribuir para o entendimento das questões relacionadas ao trabalho dos cirurgiões-dentistas e suas repercussões sobre sua vida laboral. A metodologia empregada foi baseada em entrevistas dirigidas, nas quais foram aplicados um questionário e um exame físico-funcional em cem dentistas (51 mulheres e 49 homens). Os achados indicaram que tanto homens como mulheres dentistas apresentaram problemas de origem músculo-esquelética relacionados ao trabalho em sedestração. Além disso, apesar de diferenças no método de coleta dos dados, os resultados deste estudo são compatíveis com a literatura internacional, onde os dentistas homens referem menos dores e desconforto do que as mulheres.
\end{abstract}

Palavras-chave: dentistas, risco, saúde, ergonomia

\section{Introdução}

Atualmente, as questões de saúde e organização relacionadas ao trabalho têm sido muito investigadas devido aos problemas verificados entre as diversas categorias de trabalhadores. Dentre as mais variadas atividades laborais, a odontologia se destaca por ser uma atividade voltada para a saúde, mas que impõe ao seu praticante uma série de fatores predisponentes a alterações sócio-psico-fisiológicas e organizacionais de seu trabalho.

A odontologia é uma das profíssões da área da saúde que demanda muita precisão e concentração por parte do praticante. Porém, há muitos outros fatores envolvidos na prática do dentista. Entre os principais fatores que influenciam no trabalho dos dentistas pode-se citar: a carga de trabalho psico-fisiológica e postural, em relação ao número de horas trabalhadas (RUNDCRANTZ; JOHNSSON; MORITZ, 1990) e o manejo de elementos químicos e biológicos, tais como mercúrio e sangue (BERS, 1980).

De acordo com Finsen e Christensen (1998), as posturas estáticas e o posicionamento do profissional acarretam em sobrecarga dos ombros e cervical. Isto implica em risco de desenvolver problemas ortopédicos e posturais, sendo necessário então variar as posturas e diminuir o trabalho estático, para prevenir desordens músculo-esqueléticas nos dentistas. Micholt (1990) corrobora a opinião de que o trabalho dos dentistas sobrecarrega o sistema osteomuscular destes, em especial, a coluna cervical e dorsal, ocasionando afecções ortopédicas. Desta forma, também há uma sobrecarga dos vasos sanguíneos, principalmente no que concerne ao retorno venoso ao coração.

Outro dado relevante é o maior acometimento de dores e desconforto em dentistas do sexo feminino. Rundcrantz, Johnsson e Moritz (1990, 1991b) constataram que entre dentistas mulheres a freqüência de dor e desconforto é mais alta em comparação com os dentistas homens. Além disto, estes estudos corroboraram a alta freqüência de dores de cabeça e dores e desconforto no pescoço, ombros e coluna lombar, bem como o aparecimento do absenteísmo relacionado com dor torácica e lombar. Por outro lado, os dentistas com mais idade e tempo de 
serviço, além de serem representados por homens, sofrem menos desgaste e referem menos desconforto e dores do que as mulheres em geral (RUNDCRANTZ; JOHNSSON; MORITZ, 1990). Também é relatado que as dores lombares são freqüentes tanto em homens quanto em mulheres, no entanto, as mulheres sofrem mais de lesões espinhais do que os homens, ficando mais tempo afastadas do trabalho (ANDERSSON, 1999).

Além disso, Kihara (1995) também relata problemas relacionados à atividade dos dentistas como a prevalência de problemas de visão e de membros superiores, bem como os mesmos problemas já mencionados anteriormente por Rundcrantz, Johnsson e Moritz (1990). Ekenvall, Nilsson e Falconer (1990) citam uma prevalência de problemas neuroperiféricos desenvolvidos em longo prazo pela vibração proveniente dos instrumentos utilizados pelos dentistas da ordem de $22 \%$. Há, ainda, os problemas relacionados ao posicionamento do dentista em relação ao paciente, que prejudicam o funcionamento da pinça manual responsável pela manipulação da instrumentação e a força necessária para a execução de determinadas tarefas. Neste caso, a falta ou presença de apoios para o antebraço do profissional, durante a execução do seu trabalho, influencia a intensidade de força e ou precisão necessárias para realizar a operação adequada (CATOVIC et al., 1989), assim como o local da operação de difícil acesso na boca do paciente (VIOHL apud CATOVIC et al., 1989).

Em estudos eletromiográficos, realizados por Milerad et al. (1991), também foi constatado que o músculo extensor radial do carpo da mão dominante sofre uma carga de contração estática excessiva para estabilizar o punho durante o ato de trabalho, levando à dor e ao desconforto. Da mesma forma, neste estudo ficou evidenciado que o músculo trapézio sofre sobrecarga durante o trabalho, devido a sua função de estabilizar o membro superior. $\mathrm{O}$ uso prolongado de posturas estáticas do dentista, durante seu trabalho, sugerem o aumento da pressão arterial e freqüência cardíaca em longo prazo, colocando em risco sua saúde (BOREA, 1991).

De acordo com o exposto, pode-se constatar que os dentistas em sua atividade profissional estão expostos a uma série de fatores que podem levar a acometimentos que influenciarão sua saúde e conseqüentemente a atividade profissional. Sendo assim, este estudo de caráter epidemiológico, tem como objetivo contribuir para o entendimento das questões relacionadas ao trabalho dos cirurgiões-dentistas e suas repercussões sobre a sua vida laboral, visando projetos futuros para o posto de trabalho desta categoria profissional e de forma mais ampla para a melhoria das condições de trabalho como um todo.

\section{Metodologia}

A população analisada foi determinada através do cálculo amostral baseado na população total de dentistas inscritos no Conselho Regional de Odontologia do Rio Grande do Sul, município de Porto Alegre. Esta, à época do cálculo em abril de 2003, era de 3.735 profissionais. Tal dimensionamento foi realizado levando-se em conta uma margem de erro de dez por cento com um intervalo de confiança de 95,5\%; resultando em uma amostra de no mínimo 98 sujeitos. Esta foi extrapolada para 100 sujeitos (49 homens e 51 mulheres), todos em pleno desenvolvimento de sua profissão, atendendo pacientes regularmente.

Os participantes do estudo foram selecionados aleatoriamente por convite através do jornal da categoria, bem como através de contatos telefônicos. Todos os sujeitos participaram em caráter voluntário, sendo as avaliações realizadas individualmente nos próprios consultórios dos cirurgiões-dentistas. Estas avaliações constaram de um questionário abordando 136 questões sobre aspectos psicossociais, posto de trabalho, aspectos pessoais e antecedentes músculo-esqueléticos, previamente validado. Quando da aplicação do questionário, procurando facilitar o entendimento e a familiarização do entrevistado com as perguntas, estas 
foram lidas em voz alta, sistematicamente sempre da mesma forma, respeitando de forma estrita a ordem numérica do questionário.

De maneira complementar, foi realizado um exame físico e funcional, com base no questionário elaborado por Cock e Masset (1994). O exame físico abrangeu aspectos de anamnese, como dados pessoais, além de perguntas referentes a dores em segmentos específicos, além de testes específicos de funcionalidade e palpações da musculatura dos segmentos corporais examinados.

O questionário consistiu de perguntas objetivas em valores discretos divididos nos seguintes construtos: dados pessoais, fatores músculo-esqueléticos, fatores pessoais e fatores do posto de trabalho, que buscava contemplar a avaliação dos membros superiores e coluna cervical dos entrevistados; todos antecedidos pela assinatura de um Termo de Consentimento Livre Informado.

Os dados desse estudo foram tratados pelo programa SPSS versão 10.0. Por meio dele foram levantadas as prevalências e as relações entre as variáveis do trabalho.

\section{Resultados}

Inicialmente, procurou-se caracterizar a amostra, evidenciando algumas diferenças entre sexo, com relação às características biométricas e o tempo de exposição ao trabalho da amostra analisada.

De acordo com os dados coletados, a idade média da amostra foi de 38,9 \pm 11,2 anos; peso médio de $69,8 \pm 14,5 \mathrm{~kg}$, altura média de 1,7 \pm 0,96 m e com um tempo médio de serviço de $14,9 \pm 10,1$ anos. A figura 1 ilustra os dados médios biométricos característicos totais e separados por sexo, assim como a indicação do tempo de trabalho.

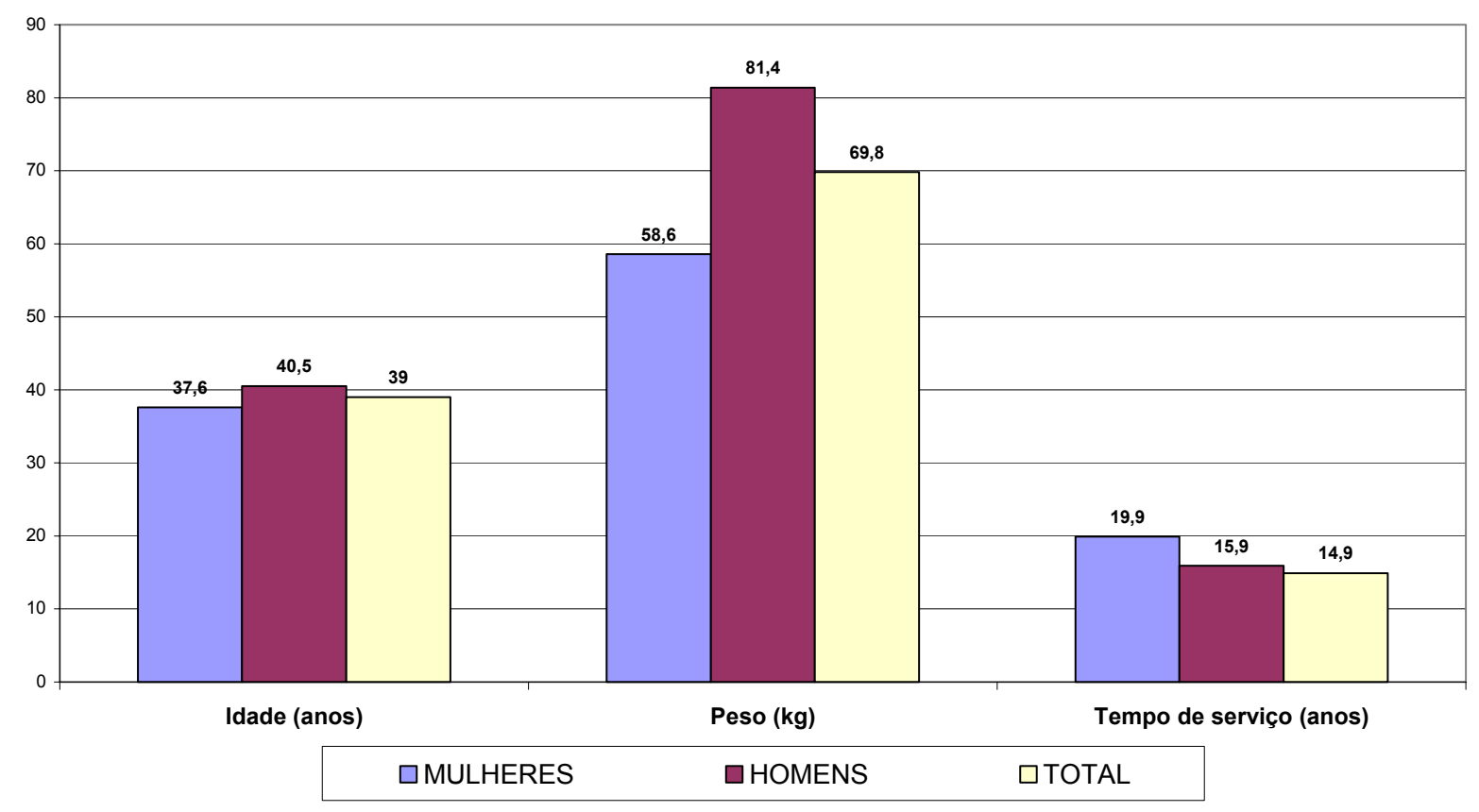

Figura 1 - Dados médios totais (biométricos e de tempo de serviço) da amostra avaliada

Com relação às características gerais psicossociais e hábitos pessoais, a tabela 1 indica as porcentagens das variáveis consideradas. Neste sentido, observou-se que mais de um quarto da amostra feminina desta pesquisa referiu ter depressão, enquanto os homens apresentaram 
uma prevalência menor de $14,3 \%$ de ocorrência de depressão. No que diz respeito às alergias, também a população feminina deste estudo apresentou uma maior ocorrência de alergias da ordem de $41,2 \%$, em comparação a $32,6 \%$ entre os homens.

Tabela 1 - Características e hábitos pessoais

\begin{tabular}{lccc}
\hline \multicolumn{1}{c}{ Variável } & Mulheres & Homens & Total \\
\hline Casados & $56,8 \%$ & $51,0 \%$ & $54,0 \%$ \\
Solteiros & $25,5 \%$ & $36,7 \%$ & $31,0 \%$ \\
Separados & $17,6 \%$ & $12,2 \%$ & $15,0 \%$ \\
Fumantes & $5,9 \%$ & $14,3 \%$ & $10,0 \%$ \\
Filhos & $52,9 \%$ & $49,0 \%$ & $53,0 \%$ \\
Prática de esportes & $51,0 \%$ & $67,3 \%$ & $59,0 \%$ \\
Instrumento musical & $21,5 \%$ & $18,0 \%$ & $20,0 \%$ \\
Cansaço físico & $80,4 \%$ & $69,4 \%$ & $75,0 \%$ \\
Dificuldade de memória & $51,0 \%$ & $51,0 \%$ & $51,0 \%$ \\
Dificuldade em adormecer & $17,4 \%$ & $22,4 \%$ & $20,0 \%$ \\
Dores de cabeça & $49,0 \%$ & $32,6 \%$ & $41,0 \%$ \\
Uso de medicamentos & $64,7 \%$ & $30,6 \%$ & $48,0 \%$ \\
Alergias & $41,2 \%$ & $32,6 \%$ & $37,0 \%$ \\
Consumo de álcool & $35,3 \%$ & $71,4 \%$ & $53,0 \%$ \\
Depressão & $25,5 \%$ & $14,3 \%$ & $20,0 \%$ \\
Uso de óculos & $52,9 \%$ & $59,2 \%$ & $56,0 \%$ \\
\hline
\end{tabular}

A tabela 2 ilustra que, entre o total dos dentistas, a grande maioria 91\% são destros. Pela tabela, também ficou evidenciado que tanto mulheres como homens adotam a postura sentada, respectivamente $90,2 \%$ e $81,6 \%$, com um total de $86 \%$ da amostra estudada adotando esta mesma forma de trabalho. Já o uso de Equipamentos de Proteção Individual (EPI) é uma unanimidade entre as mulheres, sendo que $95,9 \%$ dos homens fazem uso destes equipamentos. A carga física do trabalho de cirurgião-dentista nesta pesquisa foi mais ressentida pelas mulheres $(82,3 \%)$, do que pelos homens $(77,5 \%)$, sendo que $80 \%$ do total do estudo alegam ter uma carga de trabalho penosa. Entre as mulheres, o contato com o mercúrio durante a atividade laboral foi constatado com aproximadamente $51 \%$ das entrevistadas. Com relação a este mesmo item, os homens mencionaram contato com mercúrio durante o trabalho um pouco menor $(44,9 \%)$, e um total para a amostra que não atingiu os $50 \%$ de toda a população estudada.

Tabela 2 - Características profissionais

\begin{tabular}{lccc}
\hline \multicolumn{1}{c}{ Variável } & Mulheres & Homens & Total \\
\hline Destros & $92,1 \%$ & $89,8 \%$ & $91,0 \%$ \\
Trabalho em sedestração & $90,2 \%$ & $81,6 \%$ & $86,0 \%$ \\
Contato com mercúrio & $51,0 \%$ & $44,9 \%$ & $48,0 \%$ \\
Carga física no trabalho & $82,3 \%$ & $77,5 \%$ & $80,0 \%$ \\
Trabalho monótono & $45,1 \%$ & $42,9 \%$ & $44,0 \%$ \\
Trabalho cansativo & $86,3 \%$ & $83,7 \%$ & $85,0 \%$ \\
Mocho bem adaptado & $70,6 \%$ & $61,2 \%$ & $66,0 \%$ \\
Equipo bem adaptado & $74,5 \%$ & $79,6 \%$ & $77,0 \%$ \\
Instrumental bem adaptado & $96,1 \%$ & $95,9 \%$ & $96,0 \%$ \\
Uso de EPI & $100,0 \%$ & $95,9 \%$ & $98,0 \%$ \\
Criatividade no trabalho & $78,4 \%$ & $89,8 \%$ & $84,0 \%$ \\
Dinamismo no trabalho & $84,3 \%$ & $91,8 \%$ & $88,0 \%$ \\
Desafio no trabalho & $92,1 \%$ & $100,0 \%$ & $96,0 \%$ \\
Valorização no trabalho & $94,1 \%$ & $98,0 \%$ & $96,0 \%$ \\
\hline
\end{tabular}


De maneira complementar, a aplicação do exame físico permitiu evidenciar os fatores mais relevantes em relação à constatação de acometimentos no aparelho osteomuscular (figura 2).

$\mathrm{Na}$ avaliação de contraturas na cintura escapular, as mulheres apresentam um percentual de $82,3 \%$ de acometimentos, enquanto nos homens apenas $34,7 \%$, com um total de $59 \%$ de acometidos em toda amostra. Por outro lado, os homens são mais afetados pela lombalgia, com uma prevalência de $53,1 \%$. Neste quesito, as mulheres apresentam uma diferença para os homens de 6 pontos percentuais a menos, com um total de $50 \%$ da população estudada acusando acometimentos de lombálgicos. Na avaliação dos membros superiores, um fator considerado no estudo de prevalências foi a presença de dor espontânea nos punhos. Esta avaliação mostrou que, tanto no punho direito quanto no esquerdo, as mulheres apresentaram valores percentuais mais altos que os homens, em uma razão de três vezes mais acometimentos que os homens. Neste fator, as mulheres obtiveram uma prevalência de $15,7 \%$ e $7,8 \%$, respectivamente para o punho direito e esquerdo, enquanto os homens apresentaram os índices de $4,1 \%$ (punho direito) e $2 \%$ (punho esquerdo), sendo que a prevalência total de acometimentos de dor espontânea para cada punho foi de $10 \%$ à direita e $5 \%$ à esquerda.

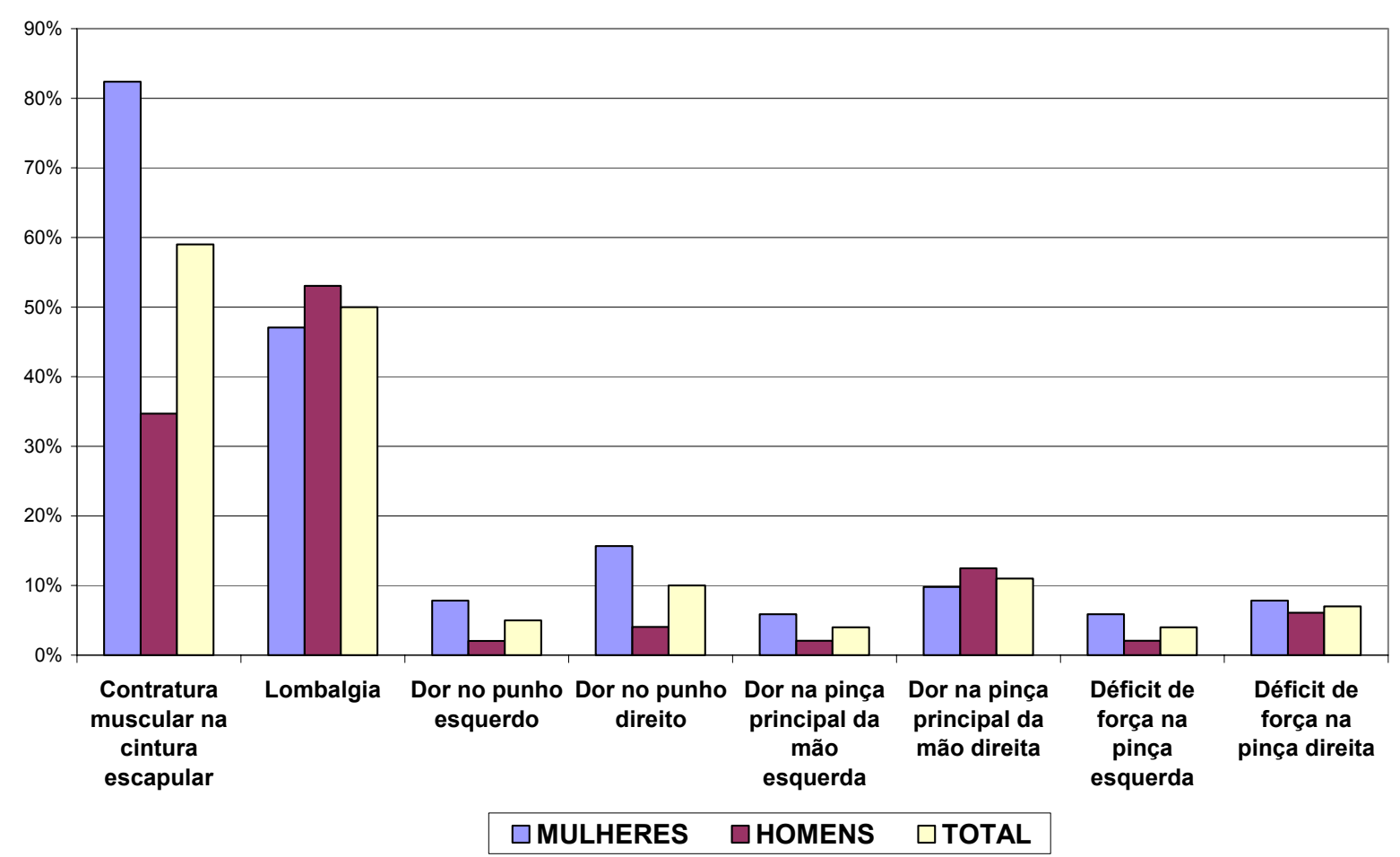

Figura 2 - Prevalências de acometimentos verificados no exame físico

A análise dos dados referentes à diferença entre sexos indicou que, do ponto de vista idade, não foram encontradas diferenças significativas. No entanto, no que concerne à altura e ao peso, o teste $t$ indicou diferenças significativas $(p<0,001)$ para ambas as variáveis.

Dessa forma, por serem fatores capazes de influir na atividade laboral dos dentistas, buscou-se evidenciar mais detalhadamente as diferenças ressentidas por ambos os sexos com relação aos itens do questionário e do exame funcional.

Assim, pela análise do teste de $\chi 2$, a tabela 3 , indica-se as diferenças significativas encontradas entre sexos considerando todos aos itens avaliados (características psicossociais e exame físico). 


\begin{tabular}{ll}
\hline Item Questionado & $\boldsymbol{p}$ \\
\hline Consumo de álcool & 0,001 \\
Uso de medicamentos & 0,001 \\
Dor à palpação na cervical & 0,017 \\
Contraturas na cintura escapular & 0,001 \\
Dor à palpação na cintura escapular & 0,004 \\
Dor espontânea no ombro direito & 0,009 \\
Dor tendão longo do bíceps esquerdo & 0,045 \\
Dor à rotação interna do ombro direito & 0,001 \\
Dor ao desvio ulnar direito & 0,045 \\
\hline
\end{tabular}

Tabela 3 - Diferenças entre sexos segundo o teste de $\chi^{2}$ $p=p$ value

Pode-se observar que os parâmetros utilizados para analisar os itens deste estudo, conforme a tabela 3 indicam que homens e mulheres apresentam algumas diferenças fundamentais.

Os homens apresentaram hábito de consumo de bebidas alcoólicas mais elevado do que as mulheres, com diferença significativa de $p=0,001$. Fato, este, corroborado pela observação de uso de álcool duas vezes maior que o sexo feminino, conforme a tabela 1 .

No que diz respeito ao uso de medicamentos, observa-se as mesmas diferenças com o mesmo índice duas vezes maior. Somente que, neste caso, são as mulheres que fazem maior uso de medicamentos, com o dobro da freqüência encontrada junto à população masculina.

A presença de contraturas musculares no ombro direito também foi evidenciada como significativamente diferente entre os sexos, com os homens apresentando mais acometimentos que as mulheres. Já no que concerne à dor espontânea no ombro direito durante o trabalho, as mulheres apresentam um quadro álgico superior.

No tocante à dor no tendão longo do bíceps esquerdo, existe um acometimento maior entre as mulheres do que com os homens. O mesmo acontece com a dor referida na rotação interna o ombro direito $(\mathrm{p}=0,001)$.

Da mesma forma, as mulheres também apresentam dor significativamente maior do que os homens, no que diz respeito ao desvio ulnar no punho direito, durante a execução do exame físico. As mulheres, por apresentarem mais queixas, podem ter interferência com o desenvolvimento de sua atividade laboral mais acentuadamente do que os homens.

\section{Discussão e conclusão}

Os resultados encontrados neste estudo permitiram comparar certas prevalências encontradas com outras disponíveis na literatura em estudos similares.

Com relação à postura de trabalho adotada pela maioria dos cirurgiões-dentistas, a sedestra, com $86 \%$ do total da amostra não difere muito dos valores percentuais encontrados por Marshall et al. (1997), Rundcrantz, Johnsson e Moritz (1990, 1991b).

Pode-se constatar comparativamente entre os estudos de Rundcrantz, Johnsson e Moritz (1990, 1991b) e Marshall et al. (1997) que o estudo atual acompanha a tendência encontrada pelos demais autores. No estudo atual, o índice prevalente de lombalgia foi maior que os estudos comparados, com aproximadamente metade da população da amostra referindo este acometimento. Já no tocante a cervicalgia, o valor prevalente deste item se manteve entre os valores máximos e mínimos das prevalências dos trabalhos internacionais. Apesar das divergências nos índices de lombalgia e cervicalgia, a prevalência do trabalho na postura sedestra apresentou um valor muito próximo aos dados dos estudos de Rundcrantz, Johnsson e Moritz (1990, 1991b) e Marshall et al. (1997) como demonstrado na figura 3. 


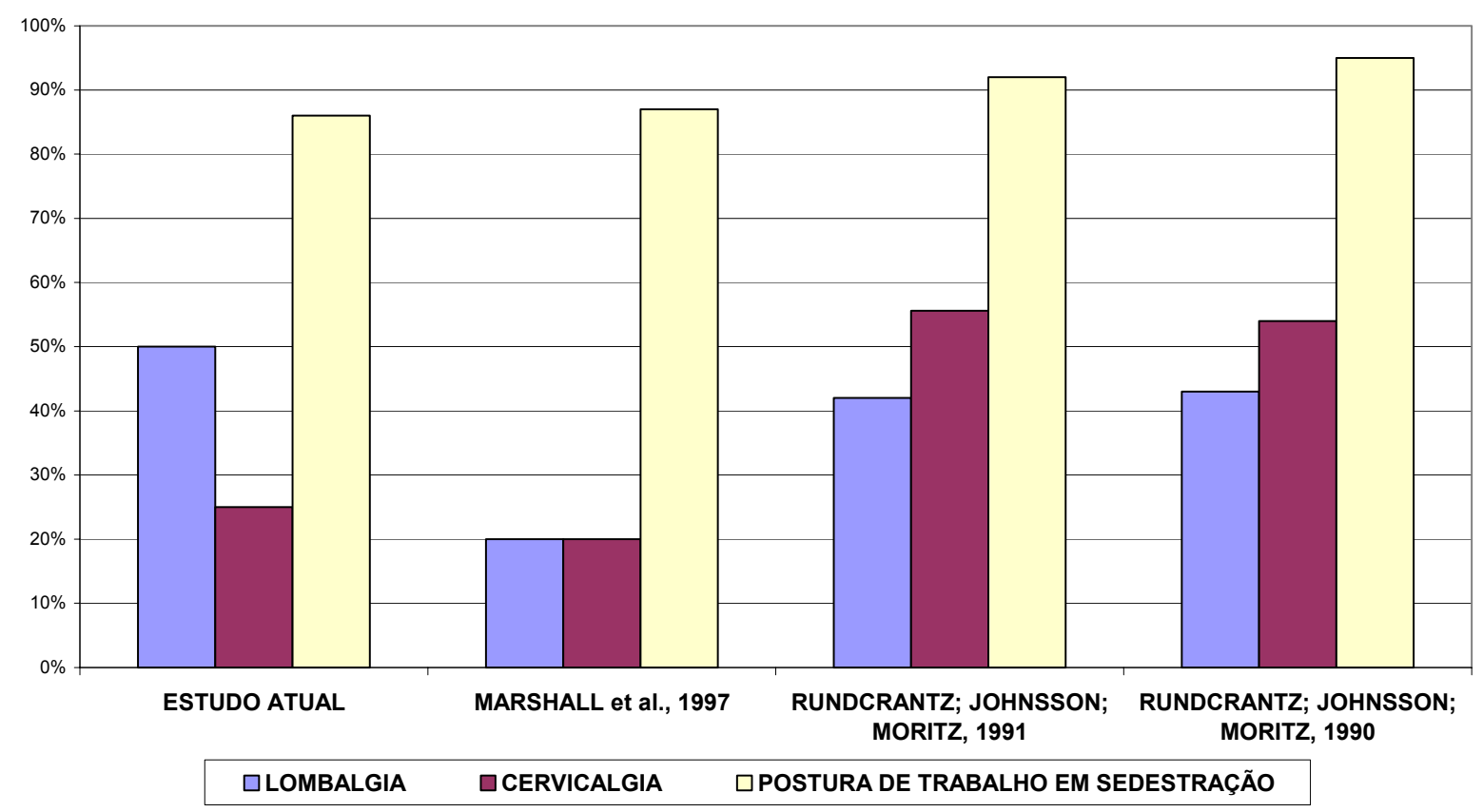

Figura 3 - Comparativo entre as prevalências da literatura e estudo atual

A atividade do cirurgião-dentista possui uma variedade de aspectos e funções, logo sua totalidade não seria possível de abranger somente neste estudo. Este se deteve a investigar a profissão como um todo. Porém, sabe-se que a odontologia é feita de várias especialidades distintas entre si, e com atividades laborais e físicas muito específicas. Essas, futuramente devem ser investigadas de forma separada, para que se possa ter uma intervenção ergonômica focalizada nas necessidades de cada especialidade. Sendo assim, trabalhos mais aprofundados em relação à postura de trabalho associada às especialidades, na organização de trabalho e sobre os equipamentos utilizados nas atividades dos odontólogos, baseados nos achados do presente estudo, podem levar a uma melhoria na qualidade da atividade laboral e de vida deste segmento profissional.

Pelo estudo realizado, fatores como o maior acometimento de lombalgias nos homens, podem ser indicativos de uma necessidade de melhor adaptação dos equipos às necessidades antropométricas, assim como a qualidade e especificidade de cada equipo, de acordo com o tipo de atividade desenvolvida e as necessidades de cada gênero. Além disso, também foi encontrada uma prevalência maior de acometimentos de dor nas mãos e punhos da população feminina deste estudo. Tal achado denota que a atividade laboral dos dentistas possui procedimentos que exigem força e habilidade no seu desenvolvimento que, com a carga de trabalho e o número de horas trabalhadas por dia, podem influenciar nas ocorrências de tais dores entre as mulheres, que aliado à dupla jornada de trabalho, pode justificar a maior prevalência de uso de medicamentos por parte da população feminina deste estudo.

Este estudo levanta dados pertinentes a futuras investigações referentes a melhorias de acessibilidade, à instrumentação e à adaptabilidade, tanto dos equipos quanto dos instrumentos de trabalho. Esta pesquisa também indica a necessidade de investigações relacionadas a aspectos organizacionais e psicossociais, baseadas nas queixas referidas e a dados comportamentais como o consumo de medicamentos e álcool por parte deste segmento profissional. 


\section{Referências}

ANDERSSON, G. B. J. (1999) - Epidemiological features of chronic low-back pain. Lancet, 354, p.581-85.

BERS, G. S. (1980) - The dentist's health. The Journal of the American Dental Association, vol. 100, Issue 4, April, p.507.

BOREA, G. (1991) - A new approach to quantify cardiovascular response in dentistry. Clin. and Exper. Hyper. - Theory and Practice, A113(4).

CATOVIC, A.; KOSOVEL, Z.; CATOVIC, E. \& MUFTIC, O. (1989) - A comparative investigation of the influence of certain arm positions on hand pinch grips in the standing and sitting positions of dentists. Applied Ergonomics, vol. 20 No. 2, p.109-114.

COCK, N. \& MASSET, D. (1994) - Le diagnostic précoce de troubles musculosquelettiques du membre supérieur en médecine du travail. Cahiers de Médecine du Travail, XXXI, 2 : 93-100.

EKENVALL, L.; NILSSON, B. Y.\& FALCONER, C. (1990) - Sensory perception in the hands of dentists. Scand. J.Work Environ. Health, No. 16, p.334-339.

FINSEN, L.; CHRISTENSEN, H. \& BAKKE, M. (1998) - Musculoskeletal disorders among dentists and variation in dental work. Applied Ergonomics, vol. 29, No. 2, p.119-125.

KIHARA, T. (1995) - Dental care and work-related complaints of dentists. The Kurume Medical Journal. Vol. 42, p.251-257.

MARSHALL, E. D.; DUNCOMBE, L. M.; ROBINSON, R. Q. \& KILBREATH, S. L. (1997) - Musculoskeletal symptoms in New South Wales dentists. Australian Dental Journal, vol. 42, No. 4, p.240-246.

MICHOLT, F. (1990) - L'ergonomie et les risques pour la santé du dentiste: vue d'ensemble. Revue Belge de Médecine Dentaire.

MILERAD, E., ERICSSON, M. O.; NISELL, R. \& KILBOM, A. (1991) - An electromyographic study of dental work. Ergonomics, vol. 34, No. 7, p. 953-962.

RUNDCRANTZ, B. L.; JOHNSSON, B \& MORITZ, U. (1990) - Cervical pain and discomfort among dentists. Epidemiological, clinical and therapeuticaspects. Part 1. A survey of pain and discomfort. Swedish Dental Journal. Volume 14, Issue 2,. p.71-80.

(1991a) - Occupational cervico-brachial disorders among dentists. Analisys of ergonomics and locomotor functions. Swedish Dental Journal, vol. 15, p.105-115.

(1991b) - Pain and discomfort in the musculoskeletal system among dentists. A prospective study.

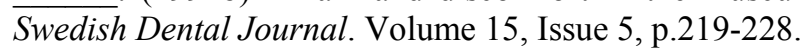

RUNDCRANTZ, B. L.; JOHNSSON, B; MORITZ, U. \& ROXENDAL, G. (1991) - Cervico-brachial disorders in dentists: a comparison between two kind of physiotherapeutic interventions. Scand. J. Rehab. Med. No. 23, p.11-17. 